\title{
NOTE ON SPELLING AND DATES
}

Throughout this work, I have modernized spelling in manuscript sources except where doing so would impede meaning or significantly alter the tone and character of a quotation. Prior to 1752, England and its colonies used the Julian calendar in which the new year began on 25 March. I have adopted dual dating, listing the Julian/Old Style year and the Gregorian/New Style year together for dates falling between 1 January and 24 March (i.e., 17 February $1606 / 1607)$. 
